\title{
Research Perspective
}

\section{Precision health for breast cancer metastasis: biomaterial scaffolds as an engineered metastatic niche to define, study, and monitor metastatic progression}

\author{
Grace G. Bushnell ${ }^{1}$, Max S. Wicha ${ }^{1}$, Jacqueline S. Jeruss ${ }^{2,3}$ and Lonnie D. Shea ${ }^{2,4}$ \\ ${ }^{1}$ Department of Internal Medicine, University of Michigan, Ann Arbor, MI, USA \\ 2 Department of Biomedical Engineering, University of Michigan, Ann Arbor, MI, USA \\ ${ }^{3}$ Department of Surgery, University of Michigan, Ann Arbor, MI, USA \\ ${ }^{4}$ Department of Chemical Engineering, University of Michigan, Ann Arbor, MI, USA \\ Correspondence to: Lonnie D. Shea, $\quad$ email: Idshea@umich.edu \\ Jacqueline S. Jeruss _jjeruss@med.umich.edu \\ Keywords: cancer metastasis; biomaterial implant; breast cancer; Hi-C; RNAseq \\ Received: July 07, $2019 \quad$ Accepted: October 01, $2019 \quad$ Published: October 09, 2019
}

Copyright: Bushnell et al. This is an open-access article distributed under the terms of the Creative Commons Attribution License 3.0 (CC BY 3.0), which permits unrestricted use, distribution, and reproduction in any medium, provided the original author and source are credited.

\section{ABSTRACT}

Metastasis represents the greatest challenge to treatment of cancer patients. Biomaterial scaffolds that recruit tumor cells to a defined site in vivo are an emerging platform for the diagnosis, treatment, and study of metastasis. Recruitment of immune cells and metastatic tumor cells to a defined location provides a precision health platform to assess current clinical cancer biomarkers in a metastatic setting, and to define the next generation of biomarkers. These platforms represent an opportunity to create a molecular staging of metastasis that could aid in both the early diagnosis and treatment of metastasis.

Metastasis represents the most common cause of death for patients with breast cancer. As a result of limitations of current technologies to detect early metastasis, they are usually not detected until they produce symptoms or are detected on scans. An emerging pre-clinical technology to detect early metastasis is a biomaterial scaffold that functions as a synthetic metastatic niche to recruit metastatic tumor cells [1]. These platforms robustly recruit tumor cells across several mouse models of metastasis [1]. Tumor cells recruited to the synthetic niche are representative of metastasis to other organs, and reflect an aggressive population; similar in metastatic ability, behavior in vitro, and transcriptome to breast cancer cells that spontaneously metastasized to the lung [2]. These findings suggest that tumor cells recruited to a synthetic niche could provide a surrogate for tumor cells in occult or relatively inaccessible locations. Additionally, the phenotype of recruited tumor cells may be reflective of disease biology and aggressiveness.

This platform technology provides a precision health platform to access clinical biomarkers expressed in the metastatic niche (Figure 1). Biomarkers are analyzed in the primary tumor for identification of subtype or proliferative index, to determine a relative risk of recurrence and treatment plan. However, for most cancers, metastases, not the primary tumor, are responsible for mortality. Tumor cell phenotypes may differ between the primary tumor and metastatic sites [3], and thus utilization of this synthetic niche provides an opportunity to gain insight into tumor biology and subtype at an accessible metastatic site. Furthermore, the synthetic niche captures early metastatic cells [4], prior to colonization of other distal tissues, and could provide a molecular staging of metastasis that guides the rational choice of therapy based on the unique biology of emerging metastases, prior to organ compromise. The synthetic niche recruits tumor cells in part through immune cell function [1] whose analysis (e.g., PD1 and PD-L1) can inform selection of immunotherapies. Emerging evidence suggests that PD1 and PD-L1 expression are differentially expressed in primary tumor and metastases [5]. Given that tumor subtype may differ between primary and metastatic disease, the differential expression of immune checkpoints would be unsurprising. Patients who have PD-L1 positive primary tumors, yet who have PD- 
L1 negative metastases, would benefit from receiving an appropriately targeted therapy while avoiding treatment delays from exposure to ineffective and potentially toxic agents. Additionally, the synthetic niche platform has potential benefits beyond enumeration of circulating tumor cells (CTCs) or disseminated tumor cells (DTCs), which has been performed as part of clinical research for more than two decades yet has not been routinely applied in clinical decision making. The presence of an easily accessible metastatic site would facilitate analysis of cells proven to be capable of metastasis [2], which distinguish them from the majority of CTCs and DTCs. Additionally, the analysis of CTCs and DTCs does not account for the microenvironment that tumor cells occupy and co-opt during the metastatic cascade. To this end, the synthetic niche recapitulates many cellular and acellular elements of the natural metastatic niche, and reflects the cellular alterations that occur systemically [6]. Collectively, the synthetic niche provides a platform for analysis of metastatic tumor cells, and the environment that influences their function and phenotype.

The synthetic niche also provides a platform to discover new biomarkers for treatments based on staging of metastasis. The future of personalized medicine will require an extension beyond the current biomarkers which have been developed based on the primary tumor. New biomarkers for metastasis are necessary as metastases are not typically detected until relatively late. In addition to detecting early metastases, the synthetic niche captures both tumor and niche stromal cells, which allows analysis of the complex interplay between tumor cells and their microenvironment from the initiation of the metastatic niche and throughout niche maturation. In contrast to vital organs, the synthetic niche can be repeatedly sampled with core biopsy without significant patient risk, and this longitudinal monitoring could be useful in both preclinical models and human clinical trials as a key feature of discovery. Gene expression and phenotypic analysis of the microenvironment, including myeloid derived suppressor cells (MDSCs), macrophages, endothelial cells, and fibroblasts, would be expected to correlate with disease progression [1]. Paracrine communication originating from stromal cells can influence epigenetic programming of the tumor cells, influencing properties such as stemness, dormancy, and resistance [7,8]. Identifying the mechanisms driving niche maturation and metastatic progression would serve as staging biomarkers and may also function as targets for therapeutic intervention aimed

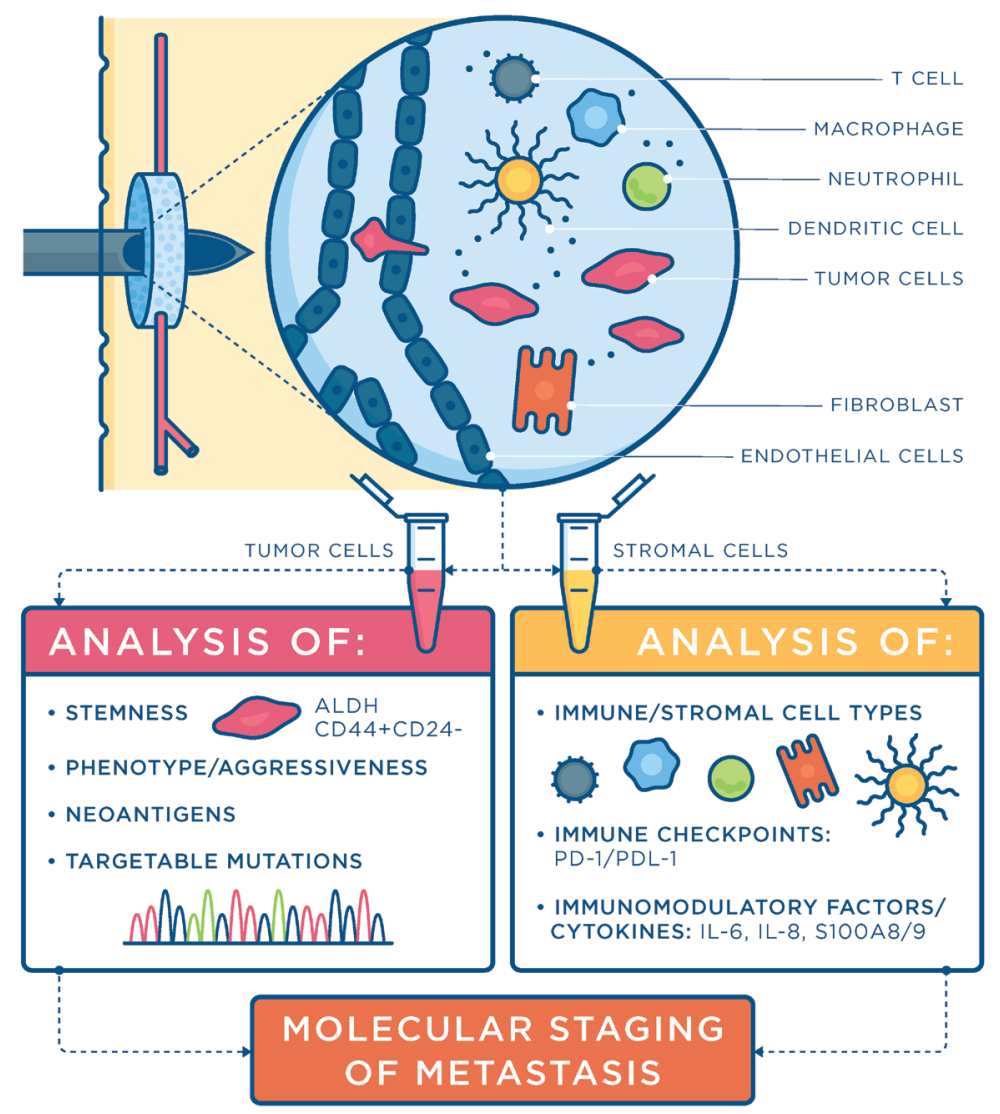

Figure 1: Engineered metastatic niche as a site to develop a molecular staging of metastasis. Biomaterial scaffolds serving as an engineered metastatic niche provide an opportunity to capture both tumor cells and metastasis associated stromal and immune cells. These cells can be analyzed using current clinical markers and may also be used to develop the next generation of metastasis biomarkers. Taken together, the engineered metastatic niche can provide a molecular staging of metastasis. 
at disrupting the microenvironmental support of metastatic tumor cells. Tumor cells recruited to the synthetic niche have characteristics similar to the native metastatic sites [2]. Interestingly, these tumor cells can be expanded [2] which could enable their use for drug screening platforms, or mutations and neoantigens may be identified that could be the basis of targeted therapeutics, or cancer vaccines, respectively [9]. This analysis of the microenvironment and tumor cells could provide the basis of a metastatic staging of disease, informing metastasis-specific therapies, and subsequent analysis of the scaffold could potentially determine sensitivity or resistance to the therapy.

In conclusion, a synthetic metastatic niche provides a pre-defined site that enables analysis of metastases from initiation to progression that is not possible due to the occult, focal, and stochastic nature of metastatic sites in vital organs. The scaffold tool is an enabling precision medicine technology to elucidate the biology and paracrine signaling that occurs at metastatic sites, which can be exploited for monitoring disease progression (or regression) and development of therapies.

\section{CONFLICTS OF INTEREST}

The scaffold as a platform for metastasis detection is described in a current patent application US20170281798A1 by assignee Northwestern University with inventors Lonnie D. Shea, Samira M. Azarin, Robert M. Gower, Jacqueline S. Jeruss and US2017012556 by assignee Regents of the University of Michigan with inventors Lonnie D. Shea, Shreyas S. Rao, Samira M. Azarin, Jacqueline S. Jeruss, and Grace G. Bushnell.

\section{FUNDING}

The authors acknowledge support from the National Institutes of Health NIH-Director's Transformative Research Award-R01CA173745 (to L.D. Shea and J.S. Jeruss). G.G. Bushnell is a recipient of the NSF Graduate Research Fellowship and NRSA F31 CA224982-01.

\section{REFERENCES}

1. Aguado, BA., Bushnell, GG., Rao, SS., Jeruss, JS., Shea LD. Engineering the pre-metastatic niche. Nat Biomed Eng. 2017;1. https://doi.org/10.1038/s41551-017-0077. [PMID:28989814]

2. Bushnell GG, Hardas TP, Hartfield RM, Zhang Y, Oakes RS, Ronquist S, Chen H, Rajapakse I, Wicha MS, Jeruss JS, Shea LD. Biomaterial Scaffolds Recruit an Aggressive Population of Metastatic Tumor Cells In Vivo. Cancer Res. 2019; 79:2042-2053. https://doi.org/10.1158/0008-5472. CAN-18-2502. [PMID:30808673]

3. Yeung C, Hilton J, Clemons M, Mazzarello S, Hutton B, Haggar F, Addison CL, Kuchuk I, Zhu X, Gelmon $\mathrm{K}$, Arnaout A. Estrogen, progesterone, and HER2/neu receptor discordance between primary and metastatic breast tumours-a review. Cancer Metastasis Rev. 2016; 35:427-37. https://doi.org/10.1007/s10555-016-9631-3. [PMID:27405651]

4. Azarin SM, Yi J, Gower RM, Aguado BA, Sullivan ME, Goodman AG, Jiang EJ, Rao SS, Ren Y, Tucker SL, Backman V, Jeruss JS, Shea LD. In vivo capture and labelfree detection of early metastatic cells. Nat Commun. 2015; 6:8094. https://doi.org/10.1038/ncomms9094. [PMID:26348915]

5. Cimino-Mathews A, Thompson E, Taube JM, Ye X, Lu Y, Meeker A, Xu H, Sharma R, Lecksell K, Cornish TC, Cuka N, Argani P, Emens LA. PD-L1 (B7-H1) expression and the immune tumor microenvironment in primary and metastatic breast carcinomas. Hum Pathol. 2016; 47:52-63. https://doi. org/10.1016/j.humpath.2015.09.003. [PMID:26527522]

6. Rao SS, Bushnell GG, Azarin SM, Spicer G, Aguado BA, Stoehr JR, Jiang EJ, Backman V, Shea LD, Jeruss JS. Enhanced survival with implantable scaffolds that capture metastatic breast cancer cells in vivo. Cancer Res. 2016; 76:5209-18. https://doi.org/10.1158/0008-5472.CAN-152106. [PMID:27635043]

7. Linde N, Fluegen G, Aguirre-Ghiso JA. The Relationship Between Dormant Cancer Cells and Their Microenvironment. Adv Cancer Res. 2016;132:45-71. https://doi.org/10.1016/bs.acr.2016.07.002. [PMID: 27613129]

8. Plaks V, Kong N, Werb Z. The cancer stem cell niche: how essential is the niche in regulating stemness of tumor cells? Cell Stem Cell. 2015; 16:225-38. https://doi.org/10.1016/j. stem.2015.02.015. [PMID:25748930]

9. Ali OA, Lewin SA, Dranoff G, Mooney DJ. Vaccines combined with immune checkpoint antibodies promote cytotoxic T-cell activity and tumor eradication. Cancer Immunol Res. 2016; 4:95-100. https://doi. org/10.1158/2326-6066.CIR-14-0126. [PMID:26669718] 